\title{
EFEITO DA BAIXA TEMPERATURA NOTURNA E DO PORTA-ENXERTO NA VARIAÇÃO DIURNA DAS TROCAS GASOSAS E NA ATIVIDADE FOTOQUÍMICA DE LARANJEIRA 'VALÊNCIA'
}

\author{
DANIELA FAVERO SÃO PEDRO MACHADO², EDUARDO CARUSO MACHADO \\ RICARDO SILVERIO MACHADO ${ }^{4}$, RAFAEL VASCONCELOS RIBEIRO
}

RESUMO-A redução da fotossíntese durante o inverno pode ser induzida por noites frias, mesmo quando as condições durante o dia não são limitantes. Laranjais são formados com plantas enxertadas, sendo o porta-enxerto citrumeleiro 'Swingle' recomendado em áreas onde há ocorrência de baixas temperaturas. Todavia, os mecanismos fisiológicos pelos quais as plantas apresentam melhor crescimento e produção são pouco conhecidos. O objetivo deste estudo foi testar a hipótese de que o efeito da baixa temperatura noturna na fotossíntese de laranjeiras é dependente do porta-enxerto utilizado, com o citrumeleiro 'Swingle' (Citrus paradisi $x$ Poncirus trifoliata) induzindo maior tolerância ao frio noturno se comparado ao limoeiro 'Cravo' (Citrus limonia). Laranjeiras 'Valência'(Citrus sinensis) com seis meses, cultivadas em sacolas plásticas $(5 \mathrm{~L})$, foram expostas por $12 \mathrm{~h}$ à temperatura noturna de 20 e $8^{\circ} \mathrm{C}$. O tratamento térmico foi realizado em câmara de crescimento, sendo apenas a parte aérea das plantas expostas às distintas temperaturas. Medidas do curso diurno das trocas gasosas e da atividade fotoquímica foram realizadas em condições ambientais naturais. O resfriamento noturno causou maior redução da assimilação de $\mathrm{CO}_{2}$, da condutância estomática e da transpiração em laranjeira 'Valência' sobre limoeiro 'Cravo' quando comparado às plantas sobre 'Swingle'. Após o tratamento de frio, as eficiências máxima $\left(F_{v} / F_{m}\right)$ e operacional $\left(F_{q}\right.$ ' $\left./ F_{m}\right)$ do fotossistema II diminuíram nas plantas sobre 'Cravo' e mantiveram-se praticamente inalteradas nas plantas enxertadas em 'Swingle'. Portanto, o porta-enxerto 'Swingle' induziu maior eficiência fotossintética em condições de frio noturno às plantas de laranjeira 'Valência' quando comparado ao porta-enxerto 'Cravo'. A queda da assimilação de $\mathrm{CO}_{2}$ em plantas resfriadas foi devida à menor condutância estomática e menor eficiência aparente de carboxilação, ou seja, resultante de limitações difusivas e metabólicas. Embora, $F_{v} / F_{m} \mathrm{e} F_{q}$ ' $F_{m}$ ' em laranjeira 'Valência' sobre 'Cravo' tenham sido mais afetados pelo resfriamento em comparação às laranjeiras sobre 'Swingle', esses não contribuíram para a redução da assimilação de $\mathrm{CO}_{2}(A)$. Porém, o frio noturno causou aumento da atividade dos drenos alternativos de elétrons (aumento da relação entre o transporte aparente de elétrons e a assimilação de $\mathrm{CO}_{2}$ ), reduzindo a eficiência aparente de carboxilação de forma mais significante em 'Valência' sobre 'Cravo' do que sobre 'Swingle'. Estes resultados confirmam a hipótese de que a ocorrência de frio noturno afeta a fotossíntese de laranjeira 'Valência' sendo os efeitos do resfriamento dependentes do porta-enxerto.

Termos para indexação: Citrus limonia, Citrus paradisi x Poncirus trifoliata, Citrus sinensis, fluorescência da clorofila, fotossíntese.

\section{EFFECTS OF LOW NIGHT TEMPERATURE AND ROOTSTOCKS ON DIURNAL VARIATION OF LEAF GAS EXCHANGE RATES AND PHOTOCHEMICAL ACTIVITY OF 'VALÊNCIA'SWEET ORANGE PLANTS}

\begin{abstract}
Decreases in photosynthesis during winter season are probably caused by low night temperature, even under nonlimiting environmental conditions during the diurnal period. Citrus orchards are formed by grafted plants, being the 'Swingle' citrumelo rootstock recommended in areas with occurrence of low temperatures. However, the physiological mechanisms related to larger growth and crop yield in those plants are poorly understood. The aim of this study was to test the hypothesis that the effect of low night temperature in photosynthesis of sweet orange plants is dependent on the rootstock species, with 'Swingle' citrumelo (Citrus paradise x Poncirus trifoliata) inducing higher tolerance to overnight chilling when compared to 'Rangpur' lime (Citrus limonia) rootstock. Six-month old 'Valência' (Citrus sinensis) sweet orange plants grown in plastic bags (5 L) were exposed overnight $\left(12 \mathrm{~h}\right.$ ) to temperatures of 20 and $8^{\circ} \mathrm{C}$. The thermal treatment was carried out inside a growth chamber where only the upper plant shoots were exposed to temperature variation. Measurements of diurnal courses of leaf gas exchange and photochemical activity were taken under natural environmental conditions. Chilling night caused larger reduction on $\mathrm{CO}_{2}$ assimilation, stomatal conductance and transpiration in plants grafted on 'Rangpur' lime when compared to those onto 'Swingle' citrumelo rootstock. After the chilling treatment, maximum $\left(F_{v} / F_{m}\right)$ and operational $\left(F_{q}{ }^{\prime} / F_{m}{ }^{\prime}\right)$ efficiencies of photosystem II decreased in plants grafted on 'Rangpur' lime, but remained practically undisturbed in plants grafted on 'Swingle' citrumelo rootstock. Therefore, the 'Swingle' citrumelo rootstock increased the photosynthetic efficiency of 'Valência' sweet orange plants exposed to overnight chilling when compared to the 'Rangpur' lime. The reduction of $\mathrm{CO}_{2}$ assimilation in chilled plants was due to low stomatal conductance and low apparent carboxylation efficiency, i.e., it is a consequence of both diffusive and metabolic limitations. Although $F_{v} / F_{m}$ and $F_{q}$ ' $/ F_{m}$ ' in plants grafted on 'Rangpur' lime have been more affected by chilling in relation to plants on 'Swingle' citrumelo rootstock, those effects were not related with decreases in $\mathrm{CO}_{2}$ assimilation. Chilling night also increased the alternative electron sinks, as shown by the increasing ratio between apparent electron transport rate and $\mathrm{CO}_{2}$ assimilation and reduced apparent carboxylation efficiency of plants grafted on 'Rangpur' lime with larger intensity than in plants onto 'Swingle' citrumelo rootstock. Those results confirm the hypothesis that low night temperature affects photosynthesis of 'Valência' sweet orange plants, being the chilling effects rootstock-dependent.
\end{abstract}

Index Terms: Citrus limonia, Citrus paradisi x Poncirus trifoliata, Citrus sinensis, chlorophyll fluorescence, photosynthesis.

\footnotetext{
1(Trabalho 139-09). Recebido em: 03-06-2009. Aceito para publicação em: 13-01-2010.

${ }^{2}$ Bióloga, Ms. IAC, Progr. de Pós-graduação em Agricultura Tropical e Subtropical, C. P. 28, CEP 13012-970, Campinas - SP. E-mail: danifavero@yahoo.com.br ${ }^{3} E_{n}{ }^{\circ}$. Agr., Dr., Pesquisador científico. IAC, Centro de Pesq. e Desenv. de Ecofisiologia e Biofísica. E-mail: caruso@iac.sp.gov.br (autor correspondente),rafael@iac.sp.gov.br

${ }^{4}$ Eng $^{\circ}$. Agr., Ms. IAC, Programa de Pós-graduação em Agricultura Tropical e Subtropical. E-mail: ricardosilveriom@gmail.com
} 


\section{INTRODUÇÃO}

Laranjeiras são submetidas a grande variação estacional da disponibilidade de água, de radiação solar, de temperatura do ar e do solo em condição de campo, sendo necessários mecanismos fisiológicos que permitam o desenvolvimento das plantas mesmo em condições limitantes (GOLDSCHMIDT, 1999; MACHADO et al., 2002; RIBEIRO; MACHADO, 2007; Ribeiro et al., 2009a,b). Em tais situações, o metabolismo primário dos citros é afetado, havendo alteração da eficiência fotossintética (MACHADO et al., 2002; RIBEIRO; MACHADO, 2007).

$\mathrm{O}$ aspecto térmico no cultivo de plantas em condição de campo é essencial, haja vista que não existem técnicas de manejo que permitam o controle dessa variável ambiental. A assimilação de $\mathrm{CO}_{2} \mathrm{em}$ laranjeira durante o inverno é significativamente menor que na primavera e verão (MACHADO et al., 2002; RIBEIRO; MACHADO, 2007; RIBEIRO et al., 2009a,b). Essa redução da assimilação de $\mathrm{CO}_{2}$ durante o inverno pode ser induzida por noites frias, mesmo quando as condições ambientais durante o dia subsequente não são limitantes, como já se verificou em citros e outras espécies perenes (ALLEN et al., 2000; ALLEN; ORT, 2001; MACHADO et al., 2002). A menor fotossíntese de laranjeiras durante o inverno está relacionada à menor eficiência de carboxilação da enzima Rubisco, menor regeneração de ribulose-1,5-bisfosfato, menor abertura estomática e aumento de drenos alternativos de elétrons, se comparado ao verão (RIBEIRO et al., 2009a,b).

A manutenção da atividade fotossintética durante o inverno é necessária para garantir a manutenção do suprimento de fotoassimilados para os frutos em desenvolvimento ou mesmo para as raízes, que apresentam crescimento alternado com a parte aérea (BEVINGTON; CASTLE, 1985; RIBEIRO; MACHADO, 2007). Logo, a produtividade e o desenvolvimento das plantas dependem da capacidade de as mesmas se adaptarem às mudanças ambientais e aos estresses, por meio de mecanismos que conferem tolerância. No caso da baixa temperatura, a utilização do citrumeleiro 'Swingle' como porta-enxerto é indicada com base no bom desenvolvimento das plantas em áreas frias (POMPEU JR., 2005). Todavia, os aspectos fisiológicos relacionados a tal tolerância são pouco conhecidos.

Neste estudo, foi avaliada a hipótese de que o efeito da baixa temperatura noturna na fotossíntese de laranjeiras é dependente do porta-enxerto utilizado, com o citrumeleiro 'Swingle' induzindo maior tolerância ao frio noturno se comparado ao limoeiro 'Cravo'. O objetivo deste trabalho foi testar a hipótese descrita realizando avaliações da dinâmica diurna das trocas gasosas e da atividade fotoquímica

\section{MATERIAL E MÉTODOS}

Foram utilizadas laranjeiras 'Valência' com 6 meses de idade enxertadas sobre duas espécies de porta-enxertos: limoeiro 'Cravo' e citrumeleiro 'Swingle', plantadas em sacolas plásticas pretas perfuradas de $5 \mathrm{~L}$ contendo substrato comercial para citros. As plantas foram obtidas pelo sistema de produção de mudas certificadas e permaneceram em casa de vegetação recebendo irrigação diariamente. Mediu-se o curso diário da fotossíntese a partir de avaliações das trocas gasosas e da atividade fotoquímica sob condições naturais. Todavia, as laranjeiras 'Valência' sobre 'Cravo' e sobre 'Swingle' foram submetidas a dois tratamentos de temperatura na parte aérea durante a noite anterior $(12 \mathrm{~h}): 8^{\circ} \mathrm{C}$ (frio noturno) ou $20{ }^{\circ} \mathrm{C}$ (controle). $\mathrm{O}$ resfriamento da parte aérea das plantas foi realizado em uma câmara de crescimento (PGR 14, Conviron, Canada) ajustada, para manter a umidade relativa do ar constante $(65 \%)$ e a temperatura do ar, conforme os tratamentos mencionados.

Durante o tratamento de baixa temperatura noturna $\left(8^{\circ} \mathrm{C}\right)$, o sistema radicular das plantas foi disposto em um recipiente capaz de manter a temperatura das raízes e do substrato a $20^{\circ} \mathrm{C}$, garantindo que somente a parte aérea da planta fosse submetida ao tratamento de baixa temperatura, como ocorre no campo (ALLEN et al., 2000; ALLEN; ORT, 2001). Na manhã seguinte $(7 \mathrm{~h})$, as plantas foram transferidas para condições naturais e foi medida a fotossíntese em folhas totalmente expandidas, de aproximadamente 2 meses, em intervalos de $1 \mathrm{~h} 30$ no período compreendido entre 8 e $16 \mathrm{~h}$. A partir das dinâmicas diurnas da assimilação de $\mathrm{CO}_{2}$, foram calculados os valores diários integrados $\left(A_{i}, \mathrm{mmol}\right.$ $\left.\mathrm{m}^{-2} \mathrm{~d}^{-1}\right)$.

As medidas de trocas gasosas e atividade fotoquímica das plantas foram feitas simultaneamente com um analisador de gases por infravermelho ( $\mathrm{Li}$ 6400, Licor, EUA), equipado com um fluorômetro modulado (6400-40, Licor, EUA). A radiação fotossinteticamente ativa $(Q)$ natural, medida no início de cada horário de avaliação, foi fixada e utilizada para as demais réplicas. As variáveis medidas foram: temperatura da folha $\left(T_{f}\right)$ e diferença de pressão de vapor entre o mesófilo da folha e ar $\left(D P V_{\text {folha-ar }}\right)$, assimilação de $\mathrm{CO}_{2}(A)$, condutância estomática $\left(g_{s}\right)$, transpiração $(E)$ e concentração intercelular de $\mathrm{CO}_{2}$ $\left(C_{i}\right)$. A razão $A / C_{i}$ foi calculada, sendo indicativa da 
eficiência aparente de carboxilação (MACHADO et al., 2005).

As variáveis relacionadas à atividade fotoquímica foram estudadas a partir da medição da fluorescência da clorofila $a$, sendo consideradas a fluorescência mínima $\left(F_{o}\right)$ e máxima $\left(F_{m}\right)$, medidas após adaptação ao escuro (30 min) e fluorescência no estado de equilíbrio dinâmico $\left(F^{\prime}\right)$ e máxima $\left(F_{m}{ }^{\prime}\right)$ medidas à luz (SCHREIBER et al., 1998). A fluorescência variável no escuro e na luz foram calculadas, respectivamente, por $F_{v}=F_{m}-F_{o}$ e $F_{q}{ }^{\prime}=F_{m}{ }^{\prime}-F^{\prime}$ (Baker, 2008). Estas variáveis foram utilizadas no cálculo da eficiência quântica máxima do fotossistema II $\left(F_{\gamma} / F_{m}\right)$, da eficiência operacional do fotossistema II $\left(F_{q}{ }^{\prime} / F_{m}{ }^{\prime}\right)$ e do transporte aparente de elétrons $\left(E T R=F_{q}^{q} / F_{m}{ }_{m}\right.$, $\mathrm{x}$ $Q \times 0,5 \times 0,84)$. Para o cálculo de $E T R$, considerou-se distribuição igual de elétrons entre os fotossistemas I e II $(0,5)$ e a absorção de luz de 0,84 (Schreiber et al., 1998). A razão $E T R / A$ foi calculada conforme Ribeiro et al. (2009a,b) e representa a atividade dos drenos alternativos de elétrons.

O delineamento experimental utilizado foi inteiramente ao acaso, com parcelas subdivididas no tempo, sendo as causas de variação a temperatura noturna $\left(8\right.$ ou $20^{\circ} \mathrm{C}$ ), o porta-enxerto (limoeiro 'Cravo' e citrumeleiro 'Swingle') e o tempo (horários de medida). Os resultados foram submetidos à análise de variância (ANOVA), sendo as médias provenientes de cinco repetições de uma folha cada. Quando encontradas diferenças significativas, as médias foram comparadas pelo teste de Tukey, a 5 $\%$ de probabilidade.

\section{RESULTADOS}

A temperatura das folhas foi ao redor de $1{ }^{\circ} \mathrm{C}$ mais elevada nas plantas submetidas ao tratamento de frio noturno $\left(8^{\circ} \mathrm{C}\right)$ em relação ao controle $(20$ ${ }^{\circ} \mathrm{C}$ ), acarretando também em $D P V_{\text {folha-ar }}$ mais elevada (Figura 1B e C). Os horários de maior disponibilidade energética ocorreram ao redor do meio dia, quando $Q$ atingiu $1.100 \mu \mathrm{mol} \mathrm{m}^{-2} \mathrm{~s}^{-1}$ (Figura 1A). Pequenas diferenças de temperatura foliar $\left(T_{f}\right)$ foram observadas nas plantas submetidas aos tratamentos térmicos noturnos, sendo os maiores valores absolutos entre 9 e $14 \mathrm{~h}$ notados nas plantas que passaram por resfriamento (Figura 1C). Todavia, essas diferenças foram suficientes para promover maior $D P V_{\text {folha-ar }}$ nas plantas resfriadas (Figura 1B).

O curso diário de $A, g_{s}$ e $E$, em ambos os tratamentos, seguiu o padrão normalmente observado para laranjeiras (RIBEIRO; MACHADO, 2007; RIBEIRO et al., 2009a) (Figura 2), ou seja, os maiores valores ocorreram na parte da manhã, decaindo com o decorrer do dia. Já no início da manhã e em praticamente todos os horários de medidas, as plantas submetidas ao frio noturno, independentemente do porta-enxerto, apresentaram decréscimo significativo de $A, g_{s}, E$ e $A / C_{i}$ em relação às plantas-controle (Figura 2). Porém, as diferenças entre os tratamentos térmicos foram mais acentuadas em 'Valência' sobre 'Cravo' quando comparada com 'Valência' sobre 'Swingle'. Este resultado diferiu daquele encontrado em mangueira e em cafeeiro (ALLEN et al., 2000; BAUER et al., 1985), em que os decréscimos dessas variáveis ocorreram somente ao redor das $12 \mathrm{~h}$ do dia seguinte à imposição do frio noturno.

Nas plantas-controle, $A_{i}$ foi maior em laranjeira sobre 'Cravo' que sobre 'Swingle' (Figura 3). Após uma noite de frio, as laranjeiras sobre os dois porta-enxertos tiveram decréscimos significativos em $A_{i}$, sendo observado maior decréscimo em plantas sobre 'Cravo' (32\%) que em 'Swingle' (22\%). Outros autores também relatam decréscimos em citros de $A$, $A_{i}$ e $g_{s}$ em laranjeiras no inverno, quando ocorrem menores temperaturas noturnas, em relação à primavera e verão, em região subtropical (MACHADO et al., 2002; RIBEIRO; MACHADO, 2007; RIBEIRO et al., 2009a, b).

Em todos os tratamentos, observam-se máximos valores de $F_{v} / F_{m}$ e $F_{q}{ }^{\prime} / F_{m}{ }^{\prime}$, decrescendo no decorrer do período diurno (Figura 4A a D). Após o resfriamento noturno, $F_{v} / F_{m}$ das plantas sobre limoeiro 'Cravo' foi significativamente menor em dois horários ( 8 e $13 \mathrm{~h}$ ), enquanto em 'Swingle' se observou redução significativa de $F_{\text {, }}$ $F_{m}$ apenas no início da manhã, entre 6 e $8 \mathrm{~h}$ (Figura 4A e B). Em 'Valência' sobre 'Cravo', a redução da temperatura noturna causou decréscimo significativo de $F_{q}{ }^{\prime} / F_{m}$ ' em praticamente todos os horários de medida, enquanto em 'Swingle' houve decréscimo significativo somente às $8 \mathrm{~h}$ (Figura $4 \mathrm{Ce} \mathrm{D}$ ). Ainda, a razão $E T R / A$, em todos os tratamentos, aumentou no decorrer do dia, sendo este aumento mais pronunciado nas plantas submetidas ao frio noturno, independentemente do porta-enxerto considerado (Figuras 4E e F).

\section{DISCUSSÃO}

Em ambos os tratamentos, houve diminuição progressiva de $A$ no decorrer do dia, que está relacionada ao fechamento parcial dos estômatos (Figuras 2C e D), o qual respondeu ao aumento de $T_{f}$ e de $D P V_{\text {folha-ar }}$ (Figuras $1 \mathrm{~B}$ e C), conforme verificado por outros autores (MEDINA; MACHADO, 1999; RIBEIRO; MACHADO, 2007). Em função das resistências associadas ao fluxo da água no sis- 
tema solo-planta-atmosfera, ocorre defasagem entre a absorção de água pelas raízes e a transpiração. Com isso, o fechamento parcial dos estômatos pode representar uma estratégia para evitar desidratação excessiva ou uma consequência de desbalanço hídrico na epiderme das folhas, levando à redução de $A$ em ambos os casos (LEVY, 1980; MACHADO et al., 2002, MEDINA; MACHADO, 1999; RIBEIRO; MACHADO, 2007; RIBEIRO et al., 2009a).

Os valores de $A$ e $g_{s}$ nas plantas submetidas ao frio noturno, foram menores se comparados às plantas-controle (Figuras 2A a D). A temperatura foliar das plantas-controle foi menor em relação às plantas submetidas ao resfriamento noturno, possivelmente devido aos maiores valores de $g$ e de $E$ (Figuras 1C a F), haja vista que a transpiração tem efeito refrigerante nas folhas. Desde que as condições de avaliação e cultivo foram semelhantes em ambos os grupos de plantas, os menores valores de $g_{s}$ estão relacionados ao resfriamento noturno. De fato, o frio noturno também causou redução de $g_{s}$ em plantas de cafeeiro, videira e mangueira (ALLEN et al., 2000; Bauer Et al., 1985; FLEXAS et al., 1999).

O fechamento parcial dos estômatos nas plantas submetidas ao frio noturno pode ser devido à inibição direta do frio sobre a fotossíntese, que aumentaria a concentração intercelular de $\mathrm{CO}_{2}\left(C_{i}\right)$ (ALLEN et al., 2000). Alternativamente, o estômato poderia ser o alvo direto do resfriamento, causando o abaixamento de $C_{i}$ e levando à redução de $A$ (ALLEN; ORT, 2001; MACHADO et al., 2002). Neste estudo, os valores de $C_{i}$ foram semelhantes para as plantas submetidas ou não ao resfriamento noturno, em ambos os porta-enxertos (dados não apresentados). Embora o fechamento parcial dos estômatos cause diminuição de $C_{i}$, esta variável não variou significativamente entre os tratamentos térmicos, possivelmente devido ao decréscimo na atividade fotossintética (Figuras $2 \mathrm{G}$ e $\mathrm{H}$ ). A atividade bioquímica da fotossíntese pode ser avaliada por $A / C_{i}$ (FARQUHAR; SHARKEY, 1982), variável que apresentou significativa redução nas plantas submetidas ao frio noturno, em ambos os porta-enxertos (Figuras $2 \mathrm{G}$ e $\mathrm{H}$ ).

A entrada de $\mathrm{CO}_{2}$ no mesófilo foliar diminuiu em função do decréscimo de $g$, mas o consumo de $\mathrm{CO}_{2}$ no cloroplasto também diminuiu. Portanto, é possível que a redução em $g_{s}$ seja devida à ação direta do frio noturno sobre o estômato, causando seu fechamento, como sugeriram Allen et al. (2000). Dessa forma, a queda de $A$ e $A_{i}$ nas plantas resfriadas estaria relacionada à menor difusão do $\mathrm{CO}_{2}$ para o interior da folha, e com a redução da atividade bioquímica relacionada à fixação do $\mathrm{CO}_{2}$ (Figura 2). É importante notar que, nas plantas de laranjeira 'Valência' sobre
'Cravo' submetidas ao frio, o decréscimo nos valores de $A, A_{i}, g_{s}$ e $A / C_{i}$ foi maior que nas plantas sobre 'Swingle', indicando que este porta-enxerto induziu maior tolerância ao frio noturno.

Nas plantas sobre ambos os porta-enxertos, verificou-se que $F_{v} / F_{m}$ foi ao redor de 0,81 , sendo observados valores levemente inferiores nas plantas expostas ao frio noturno, embora esses valores não atingiram níveis que indiquem fotoinibição por efeito do frio (CRITCHLEY, 1998). A redução de $F_{v} / F_{m}$ é considerada um mecanismo de fotoproteção, diminuindo a pressão energética nos fotossistemas em condições de excesso de energia (DEMMINGADAMS; ADAMS, 1992).

A redução de $F_{q}{ }^{\prime} / F_{m}$ ' (Figura $4 \mathrm{C}$ e D) com o aumento de $Q$ (Figura $1 \mathrm{~A}$ ) reflete o aumento de quinona $\mathrm{A}\left(Q_{A}\right)$ reduzida e indica dissipação não fotoquímica da energia de excitação (Baker, 2008). Esta queda na eficiência operacional ocorreu em todos os tratamentos com aumento de $Q$, sendo mais intensa nas plantas que foram submetidas ao frio noturno. Houve maior dissipação não fotoquímica da fluorescência nas plantas resfriadas (resultado não mostrado). Este mecanismo de dissipação de energia é fotoprotetor e está relacionado com o ciclo da xantofila (DEMMING-ADAMS; DAMS, 1992), não devendo, no entanto, comprometer a assimilação de $\mathrm{CO}_{2}$ no presente caso. Mesmo com o decréscimo de $F_{q}$ ' $/ F_{m}$,' a quantidade de elétrons transportados nos fotossistemas era suficiente para suportar a fixação de $\mathrm{CO}_{2}$. Desde que as plantas resfriadas apresentaram maior $E T R / A$ e redução de $A$ (Figuras $4 \mathrm{E}$ e F), pode-se sugerir que houve incremento da utilização de elétrons em drenos alternativos à redução fotossintética do $\mathrm{CO}_{2}$, tais como a fotorrespiração. Este tipo de resposta também funciona como um mecanismo fotoprotetor e pode explicar, em parte, a queda na eficiência aparente de carboxilação $\left(A / C_{i}\right)$ (Figura $2 \mathrm{G}$ e $\left.\mathrm{H}\right)$. 


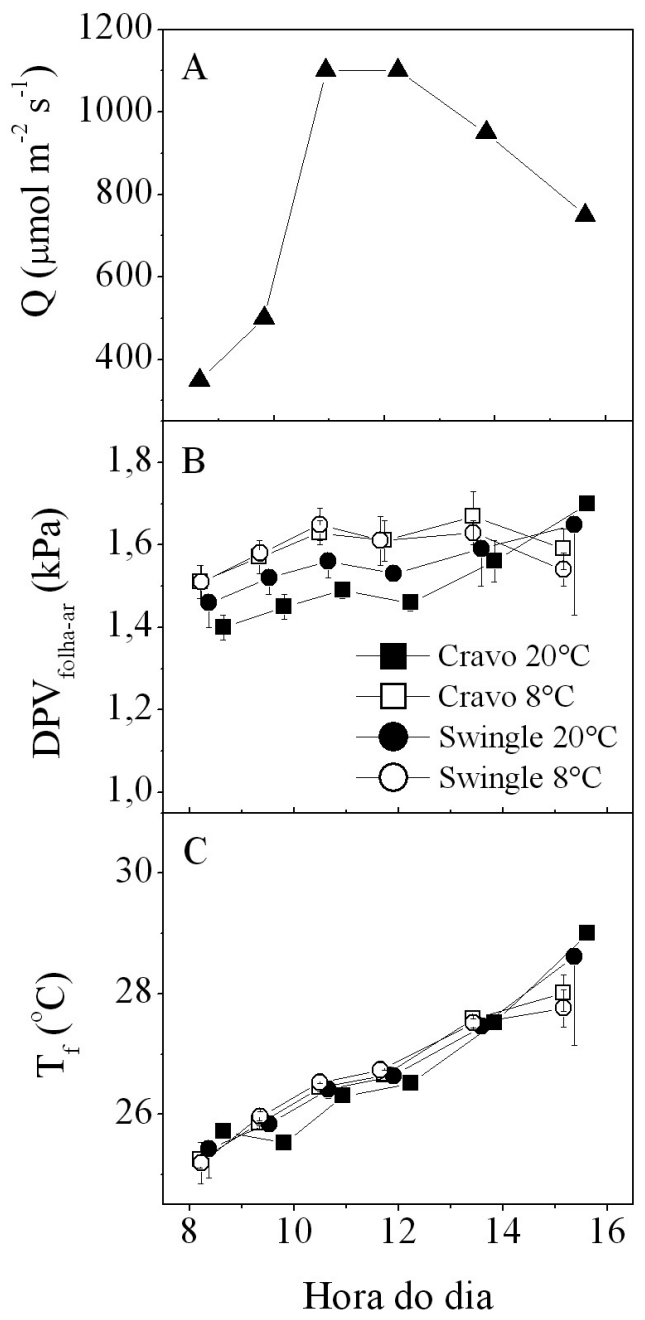

FIGURA 1 - Curso diurno da: (A) radiação fotossinteticamente ativa $(Q)$; (B) da diferença de pressão de vapor entre a folha e o ar $\left(D P V_{\text {folha-ar }}\right)$, e (C) temperatura da folha $\left(T_{f}\right) . D P V_{\text {folha-ar }}$ e $T_{f}$ foram medidos em laranjeira 'Valência' sobre limoeiro 'Cravo' $(\mathbf{\square}, \square)$ ou sobre citrumeleiro 'Swingle' $(\bullet, \circ)$, após uma noite $(12 \mathrm{~h})$ de exposição da parte aérea a $20^{\circ} \mathrm{C}(\boldsymbol{\square}, \bullet)$ ou $8{ }^{\circ} \mathrm{C}(\square, \circ)$ na parte aérea. Os símbolos representam a média de cinco repetições ( \pm desvio-padrão). 


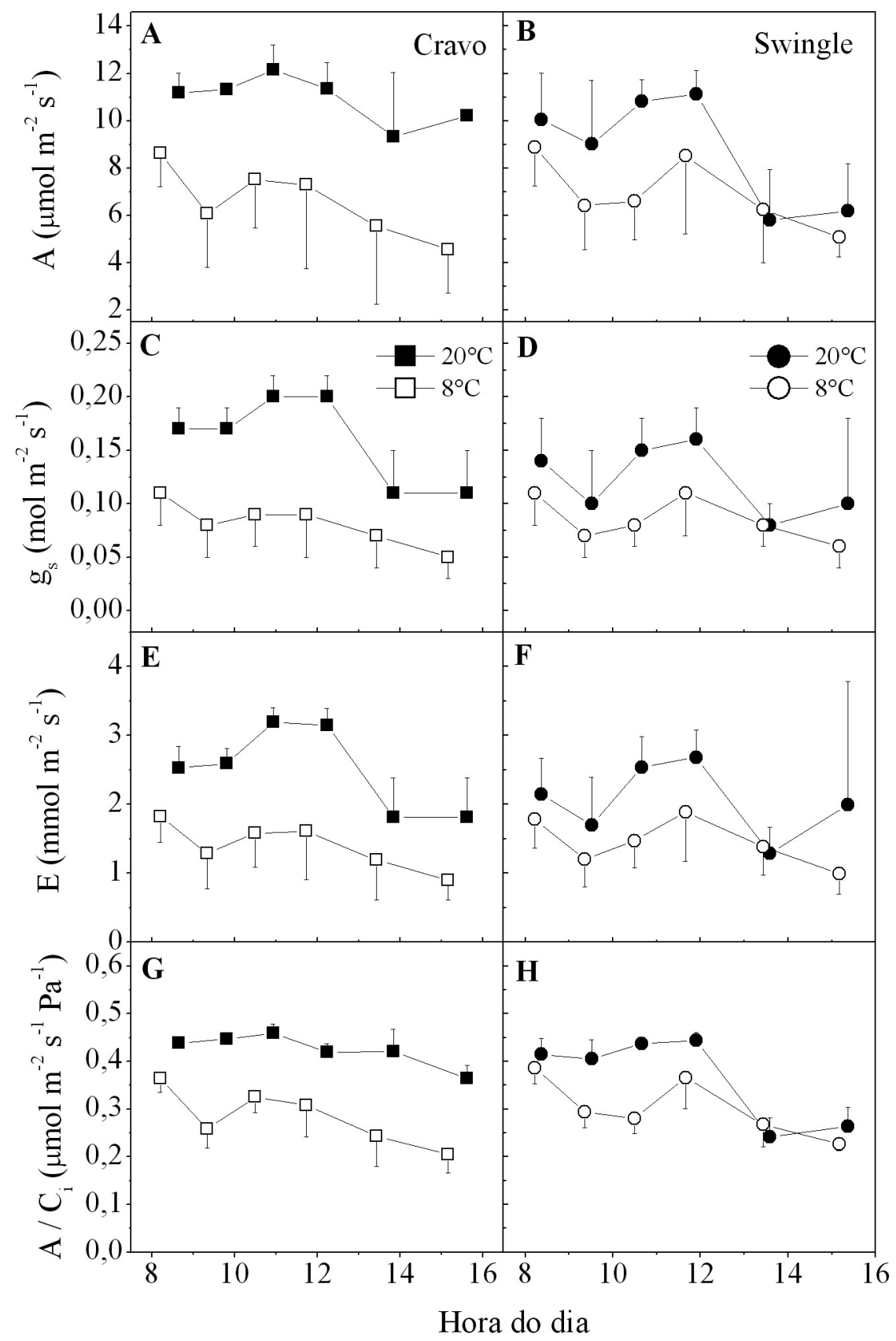

FIGURA 2 - Curso diurno da: (A e B) assimilação de CO2 (A); (C e D) condutância estomática (gs); (E e F) transpiração (E), e da (G e H) eficiência aparente de carboxilação (A/Ci) de laranjeira

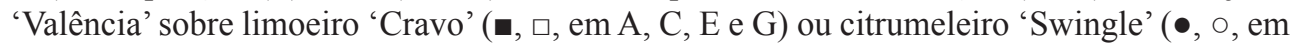
$\mathrm{B}, \mathrm{D}, \mathrm{F}$ e H), após uma noite $(12 \mathrm{~h})$ de exposição da parte aérea a $20^{\circ} \mathrm{C}(\boldsymbol{\bullet}, \bullet)$ ou $8^{\circ} \mathrm{C}(\square$, ○) na parte aérea. Os símbolos representam a média de cinco repetições ( \pm desvio-padrão). 


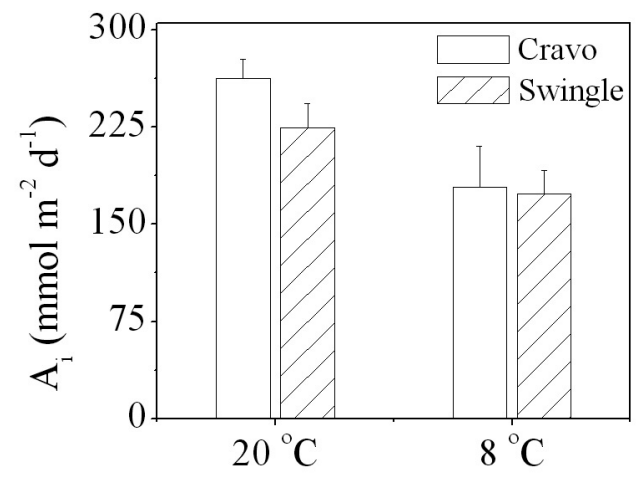

Temperatura noturna do ar

FIGURA 3 - Assimilação diurna de $\mathrm{CO}_{2}\left(A_{i}\right)$ em laranjeira 'Valência' sobre limoeiro 'Cravo' ou citrumeleiro 'Swingle', após uma noite $(12 \mathrm{~h})$ de exposição da parte aérea a 20 ou $8^{\circ} \mathrm{C}$ na parte aérea. As colunas indicam a média de cinco repetições ( \pm desvio-padrão).

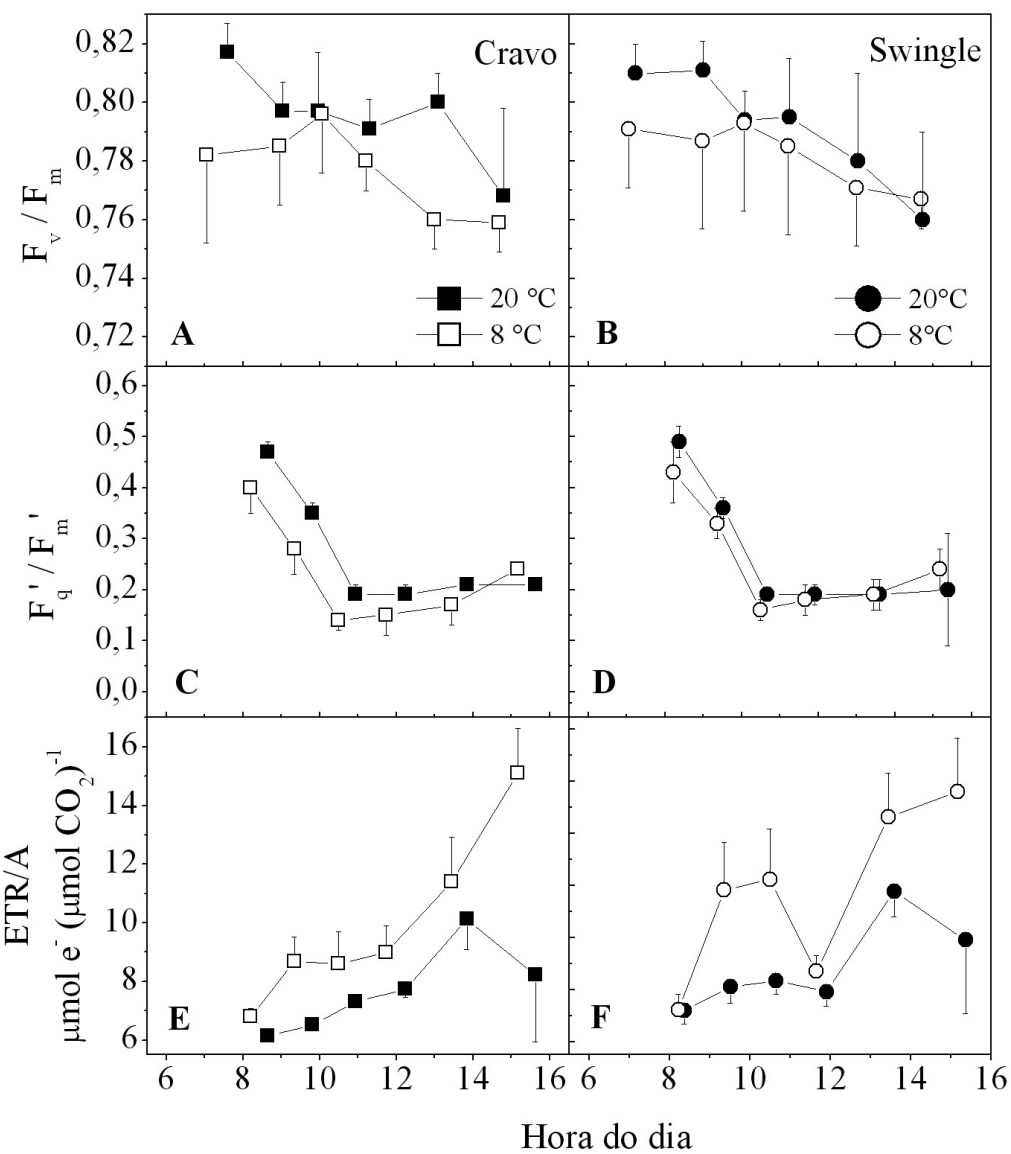

FIGURA 4 - Eficiência quântica máxima $\left(F_{v} / F_{m}\right.$, em A e B) e eficiência operacional $\left(F_{q}{ }^{\prime} / F_{m}\right.$, em C e D) do fotossistema II e relação entre o transporte aparente de elétrons e a assimilação de $\mathrm{CO}_{2}$ (ETR/A, em E e F) de laranjeira 'Valência' sobre limoeiro 'Cravo' ( $\square, \square$, em A,C,E) ou citrumeleiro 'Swingle' $(\bullet, \circ$, em B,D,F), após uma noite (12 h) de exposição da parte aérea a 20 ${ }^{\circ} \mathrm{C}(\boldsymbol{\square}, \bullet)$ ou $8{ }^{\circ} \mathrm{C}(\square, \circ)$ na parte aérea. Os símbolos indicam a média de cinco repetições $( \pm$ desvio-padrão). 


\section{CONCLUSÃO}

1-Em condições de frio noturno, plantas de laranjeira 'Valência' sobre citrumeleiro 'Swingle' apresentam menor redução da eficiência fotossintética que sobre limoeiro 'Cravo'. A exposição à baixa temperatura noturna reduz a eficiência fotossintética das plantas sobre ambos os porta-enxertos.

2-A queda da assimilação de $\mathrm{CO}_{2}$ em plantas resfriadas é devida à menor condutância estomática e menor eficiência aparente de carboxilação, ou seja, resultante de limitações difusivas e metabólicas, respectivamente. Embora, a redução de $F_{v} / F_{m}$ e $F_{q}$ ' $F_{m}$ ' em laranjeira 'Valência' sobre 'Cravo' tenha sido mais afetada pelo resfriamento em comparação às plantas sobre 'Swingle', isto não contribui para o decréscimo de $A$. No entanto, o frio noturno causa aumento da atividade dos drenos alternativos de elétrons (aumento de $E T R / A$ ), afetando a eficiência de carboxilação de forma mais significante em plantas de 'Valência' sobre 'Cravo' do que naquelas sobre 'Swingle'. Estes resultados confirmam a hipótese de que a ocorrência de frio noturno afeta a fotossíntese de laranjeira 'Valência', sendo os efeitos do resfriamento influenciados pelo porta-enxerto utilizado.

\section{AGRADECIMENTOS}

Ao Conselho Nacional de Desenvolvimento Científico e Tecnológico (CNPq), pelas bolsas a E.C.M.R.V.R. e R.S.M. e à Fundação de Apoio à Pesquisa do Estado de São Paulo (Fapesp), pelo financiamento do projeto $(05 / 57862-8)$ e pela concessão de bolsa a mestrado de D.F.S.P.M.

\section{REFERÊNCIAS}

ALLEN, D.J.; RATNER, K.; GILLER, Y.E.; GUSSAKOVSKY, E.E.; SHAHAK, Y.; ORT, D.R. An overnight chill induces a delayed inhibition of photosynthesis at midday in mango (Manguifera indica L.). Journal of Experimental Botany, Oxford, v. 51, p.1893-1902, 2000.

ALLEN, D.J.; ORT, D.R. Impacts of chilling temperatures on photosynthesis in warm-climate plants. Trends in Plant Science, London, v. 6, p. 36-42, 2001.
BAKER, N.R. Chlorophyll Fluorescence: A probe of photosynthesis in vivo. Annual Review of Plant Physiology and Plant Molecular Biology, Palo Alto, v. 59, p. 89-113, 2008.

BAUER, H.; WIERER, W.H.; HATHEWAY, H.; LARCHER, W. Photosynthesis of Coffea arabica after chilling. Physiologia Plantarum, Copenhagen, v. 64, p. 449-454, 1985.

BEVINGTON, K.B.; CASTLE, W.S. Annual roots growth pattern of young citrus trees in relation to shoot growth, soil temperature and soil water content. Journal of the American Society for Horticultural Science, Alexandria, v. 110, p. 840-845, 1985.

CRITCHLEY, C. Photoinhibition. In: RAGHAVENDRA, A.S. Photosynthesis: a comprehensive treatise. Cambridge: Cambridge University Press, 1998. chap. 20, p. 264-272.

DEMMING-ADAMS, B.; ADAMS III, W.W. Photoprotection and other responses of plants to high light stress. Annual Review of Plant Physiology and Plant Molecular Biology, Palo Alto, v. 43, p. 599-626, 1992.

FARQUHAR, G.D.; SHARKEY, T.D. Stomatal conductance and photosynthesis. Annual Review of Plant Physiology and Plant Molecular Biology, Palo Alto, v. 33, p. 317-345, 1982.

FLEXAS, J.; BADGER, M.; CHOW, W.S.; MEDRANO, H.; OSMOND, C.B. Analysis of the relative increase in photosynthetic $\mathrm{O}_{2}$ uptake when photosynthesis in grapevine leaves is inhibited following low night temperatures and/or water stress. Plant Physiology, Rockville, v. 121, p. 675-684, 1999.

GOLDSCHMIDT, E. E. Carbohydrate supply as a critical factor for citrus fruit development and productivity. HortScience, Alexandria, v. 34, p. 1020-1024, 1999.

LEVY, Y. Effect of evaporative demand on water relations of Citrus limon. Annals of Botany, Oxford, v. 46, p. $695-700,1980$. 
MACHADO, E.C.; MEDINA, C.L.; GOMES, M.M.A.; HABERMANN, G. Variação sazonal da fotossíntese, condutância estomática e potencial da água na folha de laranjeira 'Valência'. Scientia Agricola, Piracicaba, v. 59, p. 53-58, 2002.

MACHADO, E.C.; SCHMIDT, P.T.; MEDINA, C.L.; RIBEIRO, R.V. Respostas da fotossíntese a fatores ambientais em três espécies de citros. Pesquisa Agropecuária Brasileira, Brasília, v. 40, p. 1161-1170, 2005.

MEDINA, C.L.; MACHADO, E.C. Trocas gasosas e relações hídricas em laranjeira 'Valência' enxertada sobre limoeiro 'Cravo' e 'Trifoliata' e submetida à deficiência hídrica. Bragantia, Campinas, v. 57, p. 15-22, 1999.

POMPEU JR., J. Porta-enxertos. In: Mattos Jr., D.; DE NEGRI, J.D.; Pio, R.M.; POMPEU JR., J. (Ed.). Citros. Campinas: IAC/Fundag, 2005. p. 61-104.
RIBEIRO, R.V.; MACHADO, E.C. Some aspects of citrus ecophysiology in subtropical climates: re-visiting photosynthesis under natural conditions. Brazilian Journal of Plant Physiology, Londrina, v. 19, p. 393-411, 2007.

RIBEIRO, R.V.; MACHADO, E.C.; SANTOS, M.G.; OLIVEIRA, R.F. Photosynthesis and water relations of well-watered orange plants as affected by winter and summer conditions. Photosynthetica, Prague, v. 47, 2009a. No prelo

RIBEIRO, R.V.; MACHADO, E.C.; SANTOS, M.G.; OLIVEIRA, R.F. Seasonal and diurnal changes in photosynthetic limitation of young sweet orange trees. Environmental and Experimental Botany, Oxford, v. 66, 2009b. No prelo

SCHREIBER, U.; BILGER, W.; HORMANN, H.; NEUBAUER, C. Chlorophyll fluorescence as a diagnostic tool: basics and some aspects of practical relevance. In: RAGHAVENDRA, A. S. Photosynthesis: a comprehensive treatise. Cambridge: Cambridge University Press, 1998. chap. 24, p. 320-336. 